\title{
Alimentos contra el cáncer oral
}

\section{Foods against the oral cancer}

\author{
Ros Lluch N*, Chimenos Küstner E**, López López J**
}

\section{RESUMEN}

El cáncer oral representa el 5\% de todas las neoplasias y el 30\% de los cánceres de cabeza y cuello. El porcentaje de supervivencia a los 5 años es de tan sólo el $25 \%$, por lo que el diagnóstico y tratamiento precoces pueden salvar muchas vidas. La mayoría de los cánceres se relacionan con factores externos al organismo (tabaco, alcohol, betel, dieta y radiaciones solares, principalmente), que pueden modificarse o evitarse, es decir, prevenibles. Alrededor del 35\% de los casos de cáncer están relacionados con la alimentación. Por este motivo, es importante que los profesionales de la salud informen a sus pacientes acerca de las enormes posibilidades profilácticas de la dieta. El objetivo de este trabajo de actualización bibliográfica es destacar la importancia de una alimentación saludable para la prevención del cáncer oral.

Palabras clave: Cáncer oral, dieta, antioxidantes, frutas y verduras.

\section{SUMMARY}

Oral cancer means 5\% of all malignancies and 30\% of head and neck cancers. The percentage of survival at 5 years is only $25 \%$; therefore, diagnosis and early treatment can save many lives. Most cancers are related to factors outside the body (mainly tobacco, alcohol, betel, diet and solar radiation) that can be modified or avoided, i.e., they are preventable. About 35\% of cancer cases are related to food. For this reason, it is important that health professionals inform their patients about the huge prophylactic potential of diet. The aim of this work of update is to highlight the importance of a healthy diet in order to prevent the oral cancer.

Key words: Oral cancer, diet, antioxidants, fruits and vegetables.

Fecha de recepción: 5 de julio de 2008.

Aceptado para publicación: 10 de septiembre de 2008.

* Odontóloga, Diplomada en Medicina Bucal.

** Profesor Titular de Medicina Bucal. Director del Diploma de Postgrado de Medicina Bucal.

Facultad de Odontología. Universidad de Barcelona.

Ros Lluch N, Chimenos Küstner E, López López J. Alimentos contra el cáncer oral. Av. Odontoestomatol 2009; 25 (3): 155-162.

\section{INTRODUCCIÓN}

El cáncer supone un problema de salud a nivel mundial. Es una de las tres causas más importantes de mortalidad humana (Cáncer-Corazón-Carretera). El cáncer oral representa del 2 al $4 \%$ de todos los cán- ceres diagnosticados, con un incremento anual de 5.000 nuevos casos por año, aunque cabe destacar la marcada variabilidad geográfica respecto a su incidencia. Las cifras más altas las encontramos en la India y otras regiones del Sudeste asiático (representando el cáncer oral el $40 \%$ de todos los cánce- 
res orgánicos), muy relacionadas con las diferentes formas de hábito tabáquico en dichas zonas. Histológicamente, el carcinoma de células escamosas o epidermoide es el tumor maligno más común encontrado en la cavidad oral, constituyendo, aproximadamente, el 5\% de todas las neoplasias y el 30\% de los cánceres de cabeza y cuello. Aunque la cavidad oral es un área accesible y examinada con frecuencia, es muy habitual el diagnóstico tardío del carcinoma epidermoide. En estos casos, el porcentaje de supervivencia alcanza sólo el $25 \%$ a los cinco años. Es indiscutible el papel del odontólogo en la prevención primaria (promocionando un estilo de vida saludable) y secundaria (diagnóstico precoz) del cáncer oral (1).

La etiología del cáncer oral permanece desconocida. No obstante, se sabe de la existencia de una serie de factores de riesgo, que podrían actuar como agentes carcinógenos a la hora de producir el cáncer oral. La dieta, junto con el tabaco y el alcohol, se considera uno de los principales factores exógenos que contribuyen a la aparición de esta enfermedad. Se estima que alrededor del $35 \%$ de los casos de cáncer están relacionados con una dieta incorrecta. El objetivo de este trabajo es destacar la importancia de una alimentación saludable para la prevención del cáncer oral.

\section{SUSTANCIAS CANCERÍGENAS}

\section{Tabaco y alcohol}

El tabaco es el factor de riesgo más importante, observándose que casi el $90 \%$ de los pacientes con cáncer oral son fumadores. Gabriel y cols. compararon la presencia de metabolitos de folato y de indicadores de daño genético en las bocas de fumadores crónicos y no fumadores. Los resultados mostraron que el hábito crónico de fumar está asociado con una reducción sistémica del folato y de las vitaminas B6 y B12. También reduce el folato oral y provoca cambios en su forma de distribución en la boca. No obstante, el daño citológico evidente en las bocas de fumadores no está relacionado con el estado del folato oral (2). El consumo frecuente de alcohol también aumenta el riesgo de cáncer oral. Si se asocia además con el consumo de tabaco el riesgo relativo aumenta 30-50 veces. Dal Maso y cols., tras examinar una serie de estudios caso-control, llegaron a la conclusión de que, independientemente de la cantidad de alcohol consumida, los sujetos que beben entre las comidas (en ayunas) tienen un riesgo más elevado de desarrollar cáncer oral que los sujetos que beben durante las comidas. Beber con el estómago vacío puede llevar a una mayor y más rápida absorción del etanol. La ingesta de comida podría "lavar" el alcohol y reducir el efecto del etanol y de sus metabolitos carcinogénicos en la mucosa oral (3).

\section{Betel (nuez de areca)}

La marcada variabilidad geográfica de la incidencia de cáncer oral puede explicarse por la masticación de betel, hábito popular en la India y otras regiones del Sudeste asiático. Los constituyentes del betel varían en las diferentes culturas, pero normalmente consta de nuez de areca, que forma parte de la planta Piper betel (inflorescencia, hoja o raíz) y cal apagada (ya sea en forma de polvo o en pasta). En muchos países también contiene tabaco y especias, por lo que masticar betel a menudo se asocia con el consumo de tabaco. Por este motivo, hay pocos estudios que hayan estimado el papel del betel sin tabaco en la aparición del cáncer oral (4). Thomas y cols. llevaron a cabo un estudio caso-control en Papúa Guinea, donde el betel no contiene tabaco, para estudiar de forma independiente los efectos carcinogénicos del tabaco y de masticar betel. Los resultados indicaron que ambos hábitos, sobre todo el tabaco, aumentan el riesgo de padecer cáncer oral y son dosis dependientes (4).

\section{ALIMENTOS ANTICANCERÍGENOS}

\section{Frutas y verduras}

En la naturaleza, ante una agresión, los seres vegetales no pueden ni luchar ni huir. Para sobrevivir, tienen que estar provistos de unas poderosas moléculas capaces de defenderlos de las bacterias, de los insectos y de las inclemencias del tiempo. Estas moléculas son unos compuestos fitoquímicos que 
poseen propiedades antimicrobianas, antifúngicas e insecticidas, que actúan sobre los mecanismos biológicos de los posibles agresores. Además, tienen propiedades antioxidantes que protegen las células de la planta de la humedad y de los rayos del sol. Diversos estudios han demostrado que el consumo de frutas y verduras disminuye significativamente el riesgo de padecer cáncer oral. El efecto protector de estos alimentos se relaciona con múltiples componentes anticáncer, incluyendo fibra dietética, carotenoides, vitaminas $\mathrm{C}$ y $\mathrm{E}$, selenio, glucosinolados, indoles, isotiocianatos, flavonoides, polifenoles, inhibidores de la proteasa y esteroles vegetales (5). Pavia y cols. llevaron a cabo un metaanálisis para llegar a conclusiones cuantitativas sobre la contribución de la ingesta de fruta y vegetales en la existencia del cáncer oral. Según los resultados obtenidos, la ingesta de una porción de fruta al día y la ingesta de vegetales reducen significativamente el riesgo de cáncer oral en un $49 \%$ y en un $50 \%$, respectivamente. El bajo riesgo de cáncer oral asociado con el consumo de fruta está significativamente influenciado por el tipo de fruta y por el intervalo de tiempo dietético. Se observó un mayor efecto protector con el consumo de fruta cítrica y con hábitos dietéticos de tiempo prolongado (6). Boeing y cols. estudiaron la relación entre la ingesta de fruta y verdura y el riesgo de cáncer oral. Por cada porción de $80 \mathrm{~g}$ de fruta y verdura el riesgo de padecer carcinoma escamoso oral se reduce en un 9\% (12\% en los hombres; $4 \%$ en las mujeres). Este hecho apoya la iniciativa de aumentar el consumo de este tipo de alimentos (7). Chainani-Wu, tras examinar 35 estudios epidemiológicos, destaca el papel preventivo de la ingesta de vegetales (verdes, crucíferas y amarillos), de fruta, y de fruta cítrica en el desarrollo del cáncer oral. Probablemente las frutas amarillas son protectoras (8). Kreimer y cols. utilizaron los datos de una serie de estudios de caso-control realizados en nueve países para investigar los efectos de varios grupos de alimentos y del índice de masa corporal (IMC) sobre el cáncer oral. Un IMC bajo, resultado de una dieta pobre, se asoció con un elevado riesgo de cáncer oral. Los vegetales y las frutas mostraron tener un efecto protector frente al cáncer únicamente entre los usuarios fumadores y bebedores. Por lo tanto, estos grupos de alimentos pueden modular los efectos cancerígenos del tabaco y el alcohol (5). El folato es fundamental para la síntesis y reparación normal del ADN, y su déficit se asocia con un mayor riesgo de cáncer oral. Las principales fuentes de folato son las verduras de hojas verdes, las legumbres y algunas frutas. Las verduras y las frutas, especialmente los cítricos, también son ricos en flavonoides y polifenoles, que les confieren propiedades antioxidantes, antimutagénicas y antiproliferativas (5).

\section{Frutas rojas}

Las fresas, las frambuesas, los arándanos, las moras y los arándanos rojos contienen ácido elágico, así como gran cantidad de polifenoles, que estimulan los mecanismos de eliminación de sustancias cancerígenas e inhiben la angiogénesis. Además, las antocianidinas y proantocianidinas promueven la apoptosis de las células cancerosas. Rodrigo y cols. utilizaron líneas celulares humanas aisladas de tumores de carcinoma escamoso oral para investigar los efectos del extracto de etanol de frambuesa negra liofilizada sobre las características del crecimiento celular. Los resultados demostraron que el extracto de frambuesa inhibe la proliferación de las células cancerígenas, la traducción del factor de crecimiento de la citoquina angiogénica y la actividad sintasa del óxido nítrico. También induce la apoptosis y la diferenciación terminal de las células cancerígenas. Por lo tanto, es un importante agente quimiopreventivo en personas con displasia epitelial oral (9).

\section{Cítricos}

Las naranjas, las mandarinas, el limón y el pomelo, contienen flavonoides antiinflamatorios. Además estimulan la desintoxicación de sustancias cancerígenas por parte del hígado.

\section{Verduras y hortalizas ricas en carotenoides}

Las zanahorias, el ñame, la batata, el calabacín amarillo, la calabaza, ciertas variedades del calabacín naranja (conocido también como Hokkaido), el tomate y la remolacha contienen vitamina A y licopeno. El consumo de tomate y productos procesados de tomate reduce el riesgo de sufrir enfermedades 
cardiovasculares y otros procesos malignos, incluyendo el cáncer de próstata. El poder antioxidante y anticancerígeno del tomate se debe al licopeno, un pigmento vegetal de la familia de los carotenoides, que otorga al tomate su característico color rojo (10). El licopeno se asimila mejor cuando éste procede del tomate cocinado (frito, asado, zumo procesado con calor, microondas, etc.), en comparación con el tomate crudo. El aprovechamiento de esta sustancia es mayor aún si el producto se consume con un poco de grasa (aceite de oliva o de semillas) (10). Según los resultados de Allen y cols., después de 2 semanas de dieta sin licopeno, la concentración de licopeno en plasma disminuye un $50 \%$. El consumo diario de productos de tomate procesado aumenta significativamente durante dos semanas los niveles de licopeno en plasma y células de la mucosa bucal (10). Livny y cols. han demostrado, in vitro, el notable efecto anticarcinogénico del licopeno. El licopeno inhibe la proliferación de las células cancerígenas KB-1 de una manera dosis-dependiente, mediante la inhibición de la expresión de la proteína y el ARNm de la conexina 43 (11).

\section{Pescado}

Según el estudio de Kreimer y cols., el pescado también disminuye el riesgo de cáncer oral. Los pescados grasos y el aceite purificado de pescado contienen ácidos grasos poliinsaturados omega-3, que reducen la inflamación y el crecimiento de las células cancerosas (5).

\section{Té verde}

El té verde es rico en polifenoles, entre otros las catequinas (y en especial la epigalocatequina galato3 o EGCG), que reduce el crecimiento de nuevos vasos sanguíneos, necesarios para el desarrollo del tumor y para la metástasis. Es además un potente antioxidante y desintoxicante, ya que activa las enzimas del hígado que eliminan las toxinas del organismo y facilita la muerte de las células cancerosas por apoptosis. Li y cols. estudiaron en hámsters los efectos preventivos del té verde, pigmentos del té y té mixto (compuesto por extracto de agua de té verde, polifenoles y pigmentos) en el carcinógeno 7,12- dimetilbenzoantraceno o (DMBA). La administración oral de té verde, pigmentos del té y té mixto redujo significativamente el peso del tumor y la incidencia de displasia y carcinoma oral. El té mixto mostró un efecto inhibitorio del DMBA superior al de cualquier ingrediente del té (12).

\section{HÁBITOS ALIMENTARIOS}

La dieta anticáncer se compone principalmente de verduras (y legumbres) acompañadas de aceite de oliva (o de linaza) o bien de mantequilla orgánica, además de ajo, hierbas y especias. La carne y los huevos son opcionales y no representan el ingrediente principal del plato. De Stefani y cols. llevaron a cabo un estudio de caso-control para evaluar el riesgo carcinogénico de los hábitos alimenticios en Uruguay. El estofado, los alimentos ricos en grasa, el arroz, la calabaza y el boniato se asociaron con un mayor riesgo de carcinoma oral. Sin embargo, el hígado, los vegetales, los cítricos, las frutas y las legumbres se consideraron alimentos protectores. Se sugiere que las lesiones resultantes de la ingestión de alimentos muy calientes podrían dar lugar a una cadena de eventos iniciados por ulceración, hiperplasia regenerativa, displasia y finalmente carcinoma (13). Los resultados del estudio de Petridou y cols. indican que las frutas, los cereales, el aceite de oliva (y quizá los productos lácteos) protegen contra el carcinoma oral y sus efectos pueden estar mediados por un mayor consumo de riboflavina, hierro, y magnesio. Por el contrario, la carne y los productos cárnicos podrían aumentar el riesgo de cáncer (14). Los resultados del estudio de Franceschi y cols. indicaron que una elevada ingesta de vegetales, fruta, pescado y aceites vegetales, y una reducida ingesta de sopas, pasteles, carnes procesadas, huevos y mantequilla se asocian con un bajo riesgo de padecer cáncer oral (15). El consumo de embutidos como el jamón y el salami aumenta el riesgo de cáncer oral, posiblemente debido al elevado contenido de sal y nitrito o al efecto negativo de las grasas animales y del colesterol (5). Algunos estudios han encontrado que una dieta rica en carne y huevo aumenta el riesgo de cáncer oral. Estos resultados pueden estar influenciados por el tipo de grasa utilizada para freír. De hecho, en Italia, los huevos y la carne de vaca normalmente se fríen con mante- 
quilla, mientras que el pescado y las patatas se fríen con aceite, principalmente aceite de oliva y de semillas (16).

\section{MICRONUTRIENTES}

\section{Flavonoides}

Los flavonoides (del latín flavus, "amarillo") comprenden un gran grupo de componentes orgánicos naturales encontrados en una gran variedad de plantas incluyendo frutas, vegetales, nueces, semillas, cereales y té. Se calcula que en la dieta occidental se consume aproximadamente $1 \mathrm{~g}$ de flavonoides al día. Tienen un efecto antivírico, antialérgico, antiinflamatorio y anticáncer. Además, algunos flavonoides también poseen actividad antioxidante y muestran efectos sinérgicos con la vitamina C (17). La tangeritina y nobiletina son flavonoides polimetoxilados que se encuentran principalmente en la piel de la mandarina. La neohesperidina y hesperidina son los principales metabolitos secundarios de las naranjas y limones. La naringina y naringenina se encuentran en elevadas concentraciones en el pomelo (17). Miller y cols. evaluaron la actividad antineoplásica de seis flavonoides cítricos. Este experimento se realizó con hámsters, aplicando tópicamente las soluciones de los flavonoides $(2,0-2,5 \%)$ y la solución del carcinógeno 7,12-dimetilbenzoantraceno o (DMBA), en la mejilla de los roedores. Las mejillas de los controles positivos se trataron con el solvente usado para disolver los flavonoides y la solución del carcinógeno. Los datos mostraron que la hesperidina, neohesperidina, tangeritina y nobiletina fueron inactivos. En cambio, la naringina y la naringerina disminuyeron significativamente el número de tumores. La naringina también disminuyó significativamente el tamaño o peso del tumor. Los resultados sugieren que la naringina y naringenina pueden ser capaces de inhibir el desarrollo del cáncer (17). Un aspecto importante de este estudio es el hecho de que ambos flavonoides están presentes en el zumo de pomelo a concentraciones muy altas. La concentración promedio de naringina y naringenina en el zumo de pomelo blanco y rosa es de 1.000 ppm. La naringina es el compuesto más común. La proporción de naringina y naringerina normalmente supera 9:1 (17). Diversos estudios han probado que los polifenoles dietéticos, en particular los flavonoides, tienen efectos protectores frente al cáncer oral. Haghiac y cols. examinaron los efectos de la quercetina sobre el crecimiento celular, la necrosis/ apoptosis y la regulación del ciclo celular, en las células SCC-9 de un carcinoma escamoso oral humano. Según los resultados, la quercetina estimula la inhibición irreversible, dosis y tiempo dependiente, del crecimiento celular y de la síntesis de ADN. Inicialmente induce una respuesta de estrés, que provoca la necrosis de las células SCC-9, mientras que a las $72 \mathrm{~h}$ las células experimentan apoptosis, presumiblemente mediada por la inhibición de la proteína timidilato sintasa (18).

\section{Vitaminas y carotenoides}

Las vitaminas A, C, E y los carotenoides actúan como antioxidantes.

- Vitamina E (tocoferol). Es liposoluble. El $\alpha$-tocoferol es uno de los antioxidantes lipídicos más activos. Se localiza en membranas celulares y lipoproteínas, protegiéndolas de la oxidación de los radicales libres. Se encuentra en aceites de semillas (girasol, maíz, soja, oliva), frutos secos, germen de trigo, cereales integrales, yema de huevo, pescado (19).

- Vitamina C (ácido ascórbico). Es hidrosoluble. Su capacidad antioxidante se debe a su poder oxidorreductor: cede electrones a los radicales libres y los estabiliza. Regenera la forma oxidada de la vitamina $\mathrm{E}$, por lo que se aconseja ingerirlas juntas. Ejerce un efecto protector frente al tabaco. Se encuentra en el pimiento rojo, cítricos, fresones, kiwi, melón, tomate, verduras de hoja verde, crucíferas (berro, col, nabo, coliflor, brécol, rábano), hígado. La cocción destruye el 50\% de la vitamina C (19).

- Betacaroteno (provitamina A). Es liposoluble. Se convierte en el organismo en vitamina A, potente antioxidante. Se encuentra en vegetales verdes (espinaca, acelga) y amarillos (zanahoria, calabaza), crucíferas, ajo, perejil, hígados (19).

- Alfacaroteno. Con propiedades más destacadas como antioxidante que el betacaroteno, aparece en los mismos alimentos que éste aunque en una proporción menor (19). 
Según la revisión de Chainani-Wu, el caroteno, la vitamina $\mathrm{C}$ y la vitamina $\mathrm{E}$ son protectores, sobre todo cuando se combinan entre ellos y con otros micronutrientes. El papel de la vitamina A no está claro, por los resultados conflictivos de los estudios revisados (8). Maserejian y cols. evaluaron la relación entre la ingesta de vitaminas C, E y A y carotenoides y la incidencia de lesiones orales premalignas. El riesgo de lesión premaligna disminuyó significativamente en todos los pacientes, al aumentar el consumo dietético de vitamina C. Sin embargo, se observó un aumento del riesgo, sobre todo entre los fumadores, al aumentar el aporte dietético y suplementario de vitamina $\mathrm{E}$ y betacaroteno. Aunque el alfacaroteno puede reducir el riesgo de lesión precancerosa, los datos no proporcionan el apoyo suficiente para recomendar un aumento de la ingesta de este compuesto (20).

\section{MÉTODOS DE COCCIÓN}

Galeone y cols. investigaron el papel de los alimentos fritos en el cáncer oral, utilizando los datos de dos estudios de caso-control llevados a cabo en Italia y Suiza entre 1992 y 1999. En este estudio se encontró que una dieta rica en alimentos fritos se relaciona con un incremento moderado del riesgo de padecer cáncer oral (16). Durante la cocción de alimentos ricos en proteínas, como la carne o el pescado, se forman aminas heterocíclicas genotóxicas. Las variables más importantes que contribuyen a su formación son: la temperatura $\left(>150^{\circ}\right)$, el tiempo ( $>2$ min) y el método de cocción (asado en carbón vegetal, fritura y ahumado) (16).

\section{CONSIDERACIONES FINALES}

El alimento actúa todos los días, tres veces al día o más. Por tanto, tiene una considerable influencia en los mecanismos biológicos que aceleran o frenan el crecimiento del cáncer.

Todos los días, en cada comida, podemos escoger los alimentos que defenderán nuestro organismo de la invasión del cáncer. En este sentido, para prevenir el cáncer oral, debe procurarse:
- Aumentar la ingesta de:

- Frutas rojas, como las fresas, las frambuesas, los arándanos, las moras y los arándanos rojos.

- Cítricos, como las naranjas, las mandarinas, el limón y el pomelo.

- Verduras y hortalizas, en especial las ricas en carotenoides.

- Pescados grasos y aceite purificado de pescado.

- Alimentos ricos en flavonoides, como las frutas, los vegetales, las nueces, las semillas, los cereales y el té (en particular, té verde).

- Alimentos ricos en vitaminas A, C, E y carotenoides.

- Reducir o eliminar:

- Hábito tabáquico (fumado y mascado).

- Bebidas alcohólicas, especialmente en ayunas.

- Hábito de masticar betel.

- Carne y embutidos.

- Pimientos picantes, como el chile.

- Alimentos muy calientes.

- Alimentos ahumados, fritos, o asados directamente al carbón o leña.

La medicina suele actuar sobre un solo factor, esto quiere decir que los medicamentos anticáncer intervienen en una fase molecular concreta, esperando así limitar sus efectos secundarios. Los alimentos, por el contrario, actúan sobre varios mecanismos a un mismo tiempo y lo hacen suavemente, sin provocar efectos secundarios. Por esta razón, los profesionales de la salud debemos contribuir a la prevención del cáncer, formándonos previamente e informando a continuación a nuestros pacientes, acerca de las enormes posibilidades profilácticas de la dieta.

\section{BIBLIOGRAFÍA}

1. García García V, González-Moles MA, Bascones Martínez A. Bases moleculares del cáncer oral. Revisión bibliográfica. Av. Odontoestomatol 2005;21(6):287-95.

2. Gabriel HE, Crott JW, Ghandour H, Dallal GE, Choi SW, Keyes MK, Jang H, Liu Z, Nadeau M, 
Johnston A, Mager D, Mason JB. Chronic cigarette smoking is associated with diminished folate status, altered folate form distribution, and increased genetic damage in the buccal mucosa of healthy adults. Am J Clin Nutr. 2006;83(4): 835-41.

3. Dal Maso L, La Vecchia C, Polesel J, Talamini R, Levi F, Conti E, Zambon P, Negri E, Franceschi S. Alcohol drinking outside meals and cancers of the upper aero-digestive tract. Int $\mathrm{J}$ Cancer. 2002; 102(4):435-7.

4. Thomas SJ, Bain CJ, Battistutta D, Ness AR, Paissat D, Maclennan R. Betel quid not containing tobacco and oral cancer: a report on a case-control study in Papua New Guinea and a metaanalysis of current evidence. Int $\mathrm{J}$ Cancer. 2007;120(6):1318-23.

5. Kreimer AR, Randi G, Herrero R, Castellsagué X, La Vecchia C, Franceschi S; IARC Multicenter Oral Cancer Study Group. Diet and body mass, and oral and oropharyngeal squamous cell carcinomas: analysis from the IARC multinational case-control study. Int J Cancer. 2006;118(9): 2293-7.

6. Pavia M, Pileggi C, Nobile CG, Angelillo IF. Association between fruit and vegetable consumption and oral cancer: a meta-analysis of observational studies. Am J Clin Nutr. 2006;83 (5):1126-34.

7. Boeing H, Dietrich T, Hoffmann K, Pischon T y cols. Intake of fruits and vegetables and risk of cancer of the upper aero-digestive tract: the prospective EPIC-study. Cancer Causes Control. 2006;17(7):957-69.

8. Chainani-Wu N. Diet and oral, pharyngeal, and esophageal cancer. Nutr Cancer. 2002;44(2):10426.

9. Rodrigo KA, Rawal Y, Renner RJ, Schwartz SJ, Tian Q, Larsen PE, Mallery SR. Suppression of the tumorigenic phenotype in human oral squamous cell carcinoma cells by an ethanol extract derived from freeze-dried black raspberries. Nutr Cancer. 2006;54(1):58-68.

10. Allen CM, Schwartz SJ, Craft NE, Giovannucci EL, De Groff VL, Clinton SK. Changes in plasma and oral mucosal lycopene isomer concentrations in healthy adults consuming standard servings of processed tomato products. Nutr Cancer. 2003;47(1):48-56.

11. Livny O, Kaplan I, Reifen R, Polak-Charcon S, Madar Z, Schwartz B. Oral cancer cells differ from normal oral epithelial cells in tissue like organization and in response to lycopene treatment: an organotypic cell culture study. Nutr Cancer. 2003;47(2):195-209.

12. Li N, Han C, Chen J. Tea preparations protect against DMBA-induced oral carcinogenesis in hamsters. Nutr Cancer. 1999;35(1):73-9.

13. De Stefani E, Boffetta P, Ronco AL, Correa P, Oreggia F, Deneo-Pellegrini H, Mendilaharsu M, Leiva J. Dietary patterns and risk of cancer of the oral cavity and pharynx in Uruguay. Nutr Cancer. 2005;51(2):132-9.

14. Petridou E, Zavras AI, Lefatzis D, Dessypris N, Laskaris G, Dokianakis G, Segas J, Douglas CW, Diehl SR, Trichopoulos D. The role of diet and specific micronutrients in the etiology of oral carcinoma. Cancer. 2002;94(11):2981-8.

15. Franceschi S, Favero A, Conti E, Talamini R, Volpe R, Negri E, Barzan L, La Vecchia C. Food groups, oils and butter, and cancer of the oral cavity and pharynx. Br J Cancer. 1999;80(3-4): 614-20.

16. Galeone C, Pelucchi C, Talamini R, Levi F, Bosetti C, Negri E, Franceschi S, La Vecchia C. Role of fried foods and oral/pharyngeal and oesophageal cancers. Br J Cancer. 2005;92(11): 2065-9.

17. Miller EG, Peacock JJ, Bourland TC, Taylor SE, Wright JM, Patil BS, Miller EG. Inhibition of oral carcinogenesis by citrus flavonoids. Nutr Cancer. 2008;60(1):69-74. 
18. Haghiac M, Walle T. Quercetin induces necrosis and apoptosis in SCC-9 oral cancer cells. Nutr Cancer. 2005;53(2):220-31.

19. Chimenos E. Aspectos prácticos en la prevención del cáncer oral. Av Odontoestomatol 2008;24(1):61-7.

20. Maserejian NN, Giovannucci E, Rosner B, Joshipura K. Prospective study of vitamins C, E, and $A$ and carotenoids and risk of oral premalignant lesions in men. Int $\mathrm{J}$ Cancer. 2007;120(5):970-7.

\section{CORRESPONDENCIA}

Dr. Eduardo Chimenos Küstner

Vía Augusta $124,1^{\circ} 3^{a}$

08006 - BCN

e-mail: 13598eck@comb.es 